\title{
Esquematización del funcionamiento tácito de un sistema de educación superior virtual en México
}

Outlining of the tacit operation of a virtual higher education system in Mexico

Schématisation du fonctionnement tacite d'un système $d^{\prime}$ 'éducation supérieure virtuelle au Mexique

Sandra Luz García Sánchez sandraluzgarcias@gmail.com Universidad Vasco de Quiroga Morelia - México

Doctora en Ciencias Administrativas Universidad Vasco de Quiroga, Facultad de Contaduría Pública y Administración.

Rosa Amalia Gómez Ortíz ragomez10o@hotmail.com Instituto Politécnico Nacional México D.F.

Doctora en Ciencias Administrativas Instituto Politécnico Nacional, Escuela Superior de Comercio y Administración.

Artículo de investigación científica y tecnológica Según Clasificación Colciencias

Fecha de recepción: $14 / 03 / 2012$

Fecha de corrección: $28 / 04 / 2012$

Fecha de aprobación: 20/05/2012

\section{Resumen}

El crecimiento y desarrollo del mercado de servicios educativos virtuales de nivel superior en México no tiene una reglamentación oficial que regule los procesos y recursos necesarios para ejecutar la virtualidad académica; sin embargo, ante la necesidad de hacer funcionar los programas en esta modalidad de la mejor manera posible para responder a la demanda, las instituciones de educación superior (IES) que los ofertan han buscado e implementado diversas prácticas que parecen llevarse a cabo sólo en lo individual de cada una. Este documento presenta los resultados y conclusiones de una investigación que reunió evidencias para comprobar las similitudes y diferencias operativas entre diferentes esquemas de las IES oferentes de programas virtuales de nivel superior $y$, a través de su análisis y comparación, determinar aquellos procesos y elementos en común que permitirían esquematizar el funcionamiento tácito de un sistema de educación superior virtual en México.

Palabras clave: educación virtual, sistema para la virtualidad, servicios educativos virtuales, administración de la virtualidad académica. 


\section{Outlining of the tacit operation of a virtual higher education system in Mexico}

\section{Esquematización del funcionamiento}

tácito de un sistema de educación superior virtual en México

\section{Schématisation du fonctionnement tacite d'un système d'éducation supérieure virtuelle au Mexique}

\section{Abstract}

Growth and development of the service market of virtual higher education in Mexico does not have government regulations governing the processes and resources necessary to execute academic virtuality; however, upon the need to run programs in this modality in the best manner possible to respond to the demand, the higher education institutions (HEIs) offering them have sought and implemented diverse practices that seem to take place only individually at each of these. This document presents the results and conclusions of an investigation that gathered evidence to test the operational similarities and differences among different schemes at the HEls offering higher-level virtual programs and, through their analysis and comparison, determine those processes and elements in common that permit outlining the tacit operation of a virtual higher education system in Mexico.

Keywords: virtual education, system for virtuality, virtual educational services, administration of academic virtuality.

\section{Schématisation du fonctionnement tacite d'un système d'éducation supérieure virtuelle au Mexique}

Esquematización del funcionamiento tácito de un sistema de educación superior virtual en México

\author{
Outlining of the tacit operation of a virtual \\ higher education system in Mexico
}

\section{Résumée}

La croissance et le développement du marché des services éducatifs virtuels de niveau supérieur au Mexique n'a pas de réglementation officielle qui régule les processus et les ressources nécessaires pour exécuter la virtualité académique ; néanmoins, face au besoin de faire fonctionner de la meilleure façon possible les programmes sous cette modalité pour répondre à la demande, les institutions d'éducation supérieure (IES) qui les offrent, ont cherché et ont implémenté diverses pratiques qui paraissent s'effectuer seulement à l'intérieur de chaque institution. Ce document présente les résultats et les conclusions d'une recherche qui a réuni des évidences pour prouver les similitudes et les différences entre les différents schémas des IES qui offrent des programmes virtuels de niveau supérieur et, à travers leur analyse et comparaison, déterminer ces processus et ces éléments en commun qui permettraient de schématiser le fonctionnement tacite d'un système d’éducation supérieure virtuelle au Mexique.

Mots clef: éducation virtuelle, système pour la virtualité, services éducatifs virtuels, gestion de la virtualité académique. 


\section{Esquematización del funcionamiento tácito de un sistema de educación superior virtual en México}

\section{Introducción}

La educación virtual es un formato de enseñanza que se desprende de la educación a distancia y se basa para su ejecución en el uso de tecnologías de la información y la comunicación (TIC). La UNESCO (1998) definió a la educación virtual como un entorno de aprendizaje a través de un programa informático e interactivo con capacidad de comunicación, fruto de la convergencia de las tecnologías informáticas y de telecomunicaciones (1998), donde la integración de la academia a las formas de actividad implícitas en la sociedad del conocimiento y de la información, representan en todo el mundo, gracias a la globalización, una nueva forma de acercar la educación a la sociedad (UNESCO, 2009). Esta afirmación se sustenta al observar que la migración de la educación al ambiente virtual, ha tenido un fuerte crecimiento en los últimos 10 años, tanto en la cantidad de programas académicos e instituciones oferentes (ANUIES, 2007) como en el avance en el estudio y aplicación de su pedagogía, técnicas didácticas, recursos y materiales (García, 2008).

El formato virtual en la educación superior debe ser visto como una virtualización de los espacios funcionales universitarios, utilizando al ciberespacio para apoyar o sustituir tecnológicamente las actividades académicas y administrativas que de forma normalizada se llevan a cabo cotidianamente en el formato presencial de la educación superior. Los campus virtuales son la conceptualización de una universidad que funciona en el ciberespacio (Silvio, 1998), con todas sus responsabilidades y prerrogativas, pues resulta un símil paralelo del concepto presencial configurado físicamente. La educación superior virtual hace tangible el proceso educativo a través de espacios y herramientas abstractas, utilizando la figura del ciberespacio como representación de lo no presente (Chan, 2010) y utilizando lo que Levy (1999) llamó mediadores de la inteligencia colectiva para hacer palpable lo virtual. Las herramientas emanadas de la informática, la robótica, la creación de sistemas y la simulación son una constante tangible y sensorial que se materializa a través de algo físico como un equipo de cómputo (Lemke, 1993), lo que conduce a que la virtualidad se manifieste tangiblemente gracias a la digitalización y al internet (Sánchez, 2010).

A pesar del desarrollo del ámbito virtual en diversas áreas, incluyendo a la educación, el esquema educativo virtual en México funciona sin un marco normativo especialmente diseñado para la operación de programas de nivel superior ofertados bajo este formato, pues se utiliza la misma reglamentación para todas las modalidades, ajustado la operación de lo virtual a las normas concebidas en el contexto del siglo XX que no imaginan caminos diferentes para llegar al estudiante sino a través de los medios físicos del sistema presencial. Esto implica que los recursos académicos e infraestructurales solicitados para validar, operar y evaluar a los dos sistemas sean los mismos, sin considerar que la manera defuncionar en ambos sea logísticamente antagónica por la naturaleza física de lo presencial contra lo incorpóreo y tecnológico de lo virtual.

El crecimiento del mercado de servicios educativos virtuales de nivel superior ha hecho emerger una oferta de programas virtuales valiosos pero también otros sin sustento ni control (Rodríguez, 2006), debido a que el crecimiento de la modalidad ha respondido a las características individuales de las instituciones oferentes y no a las del sistema educativo en lo general (Rodríguez, 2006a), en circunstancias particulares y bajo su criterio individual (ANUIES, 2003); esto evidencia la necesidad de formalizar el perfil de los parámetros que determinen los recursos mínimos necesarios sobre los cuales pueda funcionar adecuada y eficientemente un programa en línea (Silvio, 2006), y en función a ello crear un marco regulatorio en torno a la modalidad virtual y sus oferentes (López, 2009); esto hace que sean puestas en las mesas de discusión las dudas sobre qué regular, cómo y para qué hacerlo (Flores, 2009). 
Para la construcción de una base normativa que refleje la realidad en la que se mueve la virtualidad académica se necesita, primero, conceptualizar la separación de ambas modalidades de enseñanza en función de sus diferentes naturalezas y, segundo, establecer un marco de referencia pertinente a la modalidad virtual para crear un modelo de operación administrativa con estándares que determinen la implementación de los recursos a partir de sus propias demandas y estructuras para operar la educación de grado.

\section{Marco referencial}

Para esta investigación se argumentó la existencia de un sistema de educación superior virtual, paralelo a lo presencial ortodoxo, como una organización compleja e influida por factores de origen tecnológico, mercadológico, paradigmático, normativo y discrecional. Estudiar a este organismo como vivo y evolutivo, requirió de una perspectiva epistemológica sistémica enfocada dentro de la vertiente del pensamiento complejo orientado hacia la gerencia como rama de las ciencias administrativas, para otorgar una sola dirección que acotara la perspectiva de estudio como el tipo de resultados que se iban a derivar de esa forma de abordarlo, la manera de procesarlos y el perfil de análisis que llevaría a la obtención de resultados para la construcción de un esquema descriptivo del funcionamiento tácito de la oferta de servicios educativos virtuales de nivel superior.

Para este estudio se consideraron dos tipologías de modelos de educación superior a distancia a manera de universidades virtuales: 1) la presentada por la ANUIES en el Plan Maestro de Educación Superior Abierta y a Distancia ( 2001), con 6 formas diferentes de ejercer la virtualidad en una IES, nombrando al modelo de acuerdo con sus características, ventajas y riesgos; 2 ) la que presenta Ricardo Tejeida (2003), basada en su interpretación sobre el trabajo de Jay Lemke, que refleja sustitutos de consumo para diferentes segmentos en este mercado de servicios educativos universitarios.

\section{Planteamiento}

A pesar de que las modalidades presencial y virtual son diferentes en sus esquemas de enseñanza y por ende en el tipo y operación de recursos que cada una requiere para funcionar, la normatividad del sistema educativo en México en el trámite de Registro de Validez Oficial de Estudios (REVOE) a las instituciones de educación superior (IES) privadas, pide los mismos tipos de requisitos físicos para ambos casos en la apertura y evaluación de programas de grado, ignorando con ello las necesidades propias de la naturaleza virtual para su ejecución. En el caso de las universidades con origen de capital público su derecho de autonomía les confiere la posibilidad de interpretar sus necesidades operativas para ejecutar la virtualidad académica de nivel superior de forma paralela a lo que puede ser determinado por la Secretaría de Educación Pública (SEP) en México.

Ante la carencia de un marco formal regulatorio, en la práctica académica ya se han implementado tácitamente recursos y funciones para operar la virtualidad académica fuera de lo meramente oficial, de una manera natural y hasta cierto punto reactiva, al dar respuesta a demandas de la educación en sus formatos emergentes, y muestra de ello es la conformación de una oferta creciente que integra diferentes tipos de universidades virtuales. En este marco de consideraciones, el planteamiento de investigación que orientó a este texto fue en México ¿cuál es el esquema de funcionamiento que implementan en la práctica las instituciones de educación superior para operar los programas académicos virtuales de nivel superior?

\section{Método}

La información que se presenta en este documento es de corte transversal, con una la recolección de datos entre junio y diciembre de 2010, y una revisión para actualización en septiembre del año 2011. Esta investigación, exploratoria y descriptiva, tuvo como meta la obtención de un esquema de funcionamiento operativo de la educación de grado virtual que integrara los procesos y elementos comunes observados en la operación, dirigida al usuario de programas virtuales de nivel superior.

Se consideró que la zona esencial de atención en una IES con oferta académica virtual, es la interfaz que se muestra en las pantallas para hacer uso de los recursos en línea, y de admitir que las variaciones entre cada una de éstas con respecto a la logística 
y procedimientos que tiene cada uno, pero verificando el cumplimiento del objetivo del vínculo en la navegación para cada caso. Así, la inmersión al ambiente se llevó a cabo a través de la observación y la recolección de datos observados en la navegación a través de las páginas oficiales de la modalidad en las IES; la muestra se realizó mediante la creación y uso de 10 instrumentos que tenían el fin de estructurar los indicadores y sub-indicadores utilizados para cada caso y la integración de registros de las páginas web institucionales de las IES seleccionadas, para obtener información externa, desde el punto de vista del usuario, de cada área de la universidad que la vinculara a los sistemas de educación virtual.

La recolección de datos se enfocó en la información aportada a través de las páginas web de una muestra de 10 instituciones mexicanas, con origen de capital público o privado, registradas en la SEP y autorizadas para impartir cursos de grado en modalidad no escolarizada en su vertiente virtual, integradas en el catálogo del Directorio Nacional de Instituciones de Educación Superior con Oferta de Programas de Nivel Superior Virtuales no Escolarizados de la Asociación Nacional de Universidades e Instituciones de Educación Superior (ANUIES) y/o a la Asociación Mexicana de Educación Continua y a Distancia (AMECYD), o sean, parte de los proyectos directamente operados por la SEP o los gobiernos de los diferentes niveles. Para esta etapa se crearon 10 instrumentos (cada uno integrado en una tabla de registro de observaciones) para trabajar la información que se obtuvo en la observación directa de las páginas de cada IES, con el fin de estructurar los indicadores y sub-indicadores de los procesos críticos.

El método general fue el analítico, pero se utilizaron tres métodos particulares en diferentes momentos de la investigación. El primer método fue el empírico para encontrar las guías iniciales que permitieran abordar la investigación pues con ellas se podían reconocer de antemano los aspectos que resultaban relevantes para los propósitos investigativos en el armado del sistema. El empirismo fundamentó la creación de las categorías abiertas al contrastar procesos a partir de la experiencia del investigador con experiencias presentadas por las universidades icónicas de la modalidad y la concepción teórica que las fundamentaba. En un segundo momento se utilizó de inicio el método comparativo como particular, lo cual permitió encontrar las similitudes y diferencias entre las IES integrantes de la muestra para encontrar los puntos de incidencia; el método sintético posibilitó la reunión de elementos que describían al esquema tácito de funcionamiento actual para la oferta de programas en un sistema virtual de educación superior. La selección de la gama de métodos se derivó de la construcción teórica de la investigación.

Las técnicas seleccionadas fueron la de observación no participante, la hermenéutica y la heurística que posibilitaron el tipo de levantamiento y procesamiento de información requerido por el estudio; por una parte, la logística para la obtención de datos, requirió de técnicas de observación no participante, pues la recolección era meramente con fines descriptivos, y como la fuente principal de recolección de datos eran documentos y páginas web de las universidades muestra, se utilizó la técnica hermenéutica. La síntesis requirió a la heurística como técnica para integrar el esquema de funcionamiento en un ejercicio de abstracción que tuvo como símil al funcionamiento estándar de una universidad presencial.

Las 10 herramientas utilizadas fueron tablas integradoras de información para registrar las observaciones de cada indicador integrado por sub-indicadores; de cada tabla se procesaron los datos para obtener información para la siguiente construcción de categorías con base en la relación de mayor frecuencia. A continuación se muestra un ejemplo de estas herramientas. (Tabla 1)

\section{Resultados de la investigación}

Los resultados de la investigación se conforman desde la categorización abierta inicial y la selección de áreas primarias que se consideraron como procesos necesarios, y que fueron depurados para obtener los necesarios que eran verdaderamente críticos para el funcionamiento y operación del programa virtual. Los resultados del análisis de indicadores y elementos de los procesos críticos compone la parte final de los resultados del estudio y sirvieron de base para la creación del esquema general de operación tácita actual de un programa educativo virtual de nivel superior, pues se obtuvieron los recursos coincidentes para formalizar el tipo de elementos que sustentaron la actividad que resultó como común denominador de las IES estudiadas. 


\begin{tabular}{|c|c|c|}
\hline No. & $\begin{array}{c}\text { Universidad } \\
\text { muestra }\end{array}$ & \multicolumn{1}{c|}{ Tipo de IES virtual } \\
\hline 1 & IES 1 & $\begin{array}{l}\text { Universidad virtual con extensión } \\
\text { universitaria presencial/Unidades al } \\
\text { interior de las instituciones con distinta } \\
\text { organización y curriculum. }\end{array}$ \\
\hline 2 & IES 2 & $\begin{array}{l}\text { Universidad virtual con extensión } \\
\text { universitaria presencial/Unidades al } \\
\text { interior de las instituciones con distinta } \\
\text { organización y curriculum. }\end{array}$ \\
\hline
\end{tabular}

3 IES 3 Universidad virtual adosada a la universidad tradicional/Unidades al interior de las instituciones compartiendo currícula, recursos y personal.

4 IES 4 Universidad virtual adosada a la universidad tradicional/Unidades al interior de las instituciones compartiendo currícula, recursos y personal.

5 IES 5 Universidad virtual adosada a la universidad tradicional/Unidades al interior de las instituciones con distinta organización y curriculum.

6 IES 6 Universidad presencial que introduce elementos de virtualidad en su dinámica educativa/Unidades al interior de las instituciones compartiendo currícula, recursos y personal.

$7 \quad \mathrm{IES}_{7}$ Universidad presencial que introduce elementos de virtualidad en su dinámica educativa/Unidades al interior de las instituciones compartiendo currícula, recursos y personal.

8 IES 8 Universidad presencial con extensión universitaria virtual/Unidades al interior de las instituciones compartiendo currícula, recursos y personal.

9 IES 9 Universidad presencial con extensión universitaria virtual/Unidades al interior de las instituciones con distinta organización y curriculum.

10 IES 10 Universidad presencial que introduce elementos de virtualidad en su dinámica educativa/Unidades al interior de las instituciones compartiendo currícula, recursos y personal.
Instalaciones físicas para atención del usuario virtual

Sede central del

programa

Oficinas

descentralizadas locales

"A través de la

infraestructurafísica

presencial"

"A través de la

infraestructurafísica

presencial"

Apoyo presencial en la modalidad

Apoyo institucional a

estudiantes vía centros de apoyo universitarios

En la URL en construcción se

observa la existencia de Centros

de acceso (mencionados pero sin vínculo ni información y no se encontró más información disponible en línea)

Red de centros de educación continua

"A través de la

infraestructurafísica

presencial"

"A través de la

infraestructurafísica

presencial"

"A través de la

infraestructurafísica

presencial"

"A través de la infraestructurafísica presencial"

A través de la infraestructura física de la universidad virtual

"A través de la infraestructurafísica presencial"
"A través de la infraestructurafísica presencial"

Centros de educación a distancia presenciales en diversos estados del país

Apoyo presencial en comunidades fuera del centro universitario

Nodos regionales

Extensiones universitarias regionales

Sedes seleccionados en México y Latinoamérica

"A través de la presencial" infraestructurafísica

\section{Fuente: García, S. L. y Gómez, R. A. (2012).}

\subsection{La determinación de los procesos necesarios}

La creación empírica de una categorización inicial dio la pauta para abordar administrativamente a la investigación. El mapa mental de la Figura 1 muestra este acercamiento e introducción al ambiente de la investigación (Figura 1).
Para validar a esta categorización se llevó a cabo una revisión a nivel conceptual de las áreas de trabajo y organigramas a través de las páginas de una selección de 4 universidades emblemáticas para el desarrollo de la educación superior virtual, como la forma de esquematizar esa parte de su funcionamiento en áreas operativas: la Universidad Nacional de Educación a Distancia (UNED) en España, la 


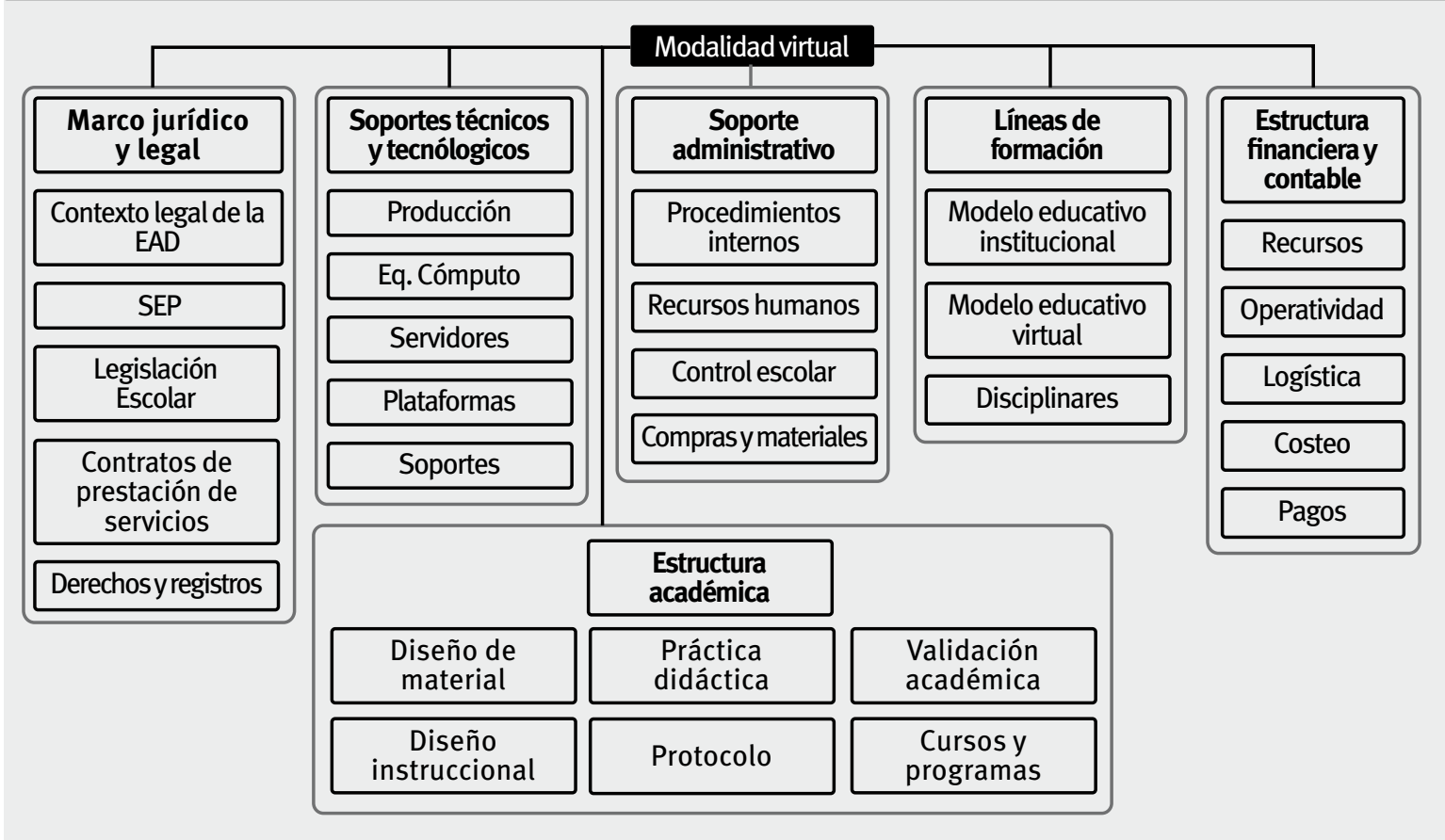

Fuente: García, S. L. y Gómez, R. A. (2012).

Universitat Oberta de Catalunya (UOC) también española, la Universidad de Guadalajara en su Sistema de Universidad Virtual (UDG Virtual) en México y The Open University en Inglaterra.

La información que se obtuvo se concentró en unificar los criterios de la categorización abierta inicial en torno a las áreas comunes de trabajo universitario, haciendo énfasis en los considerados como personajes y áreas clave para la ejecución de la modalidad; de esta manera la nueva concentración condujo a una tipología operativa general por áreas de actividad, de las cuales, en correspondencia con las diversas líneas del mapa mental anterior, surge un nuevo esquema con la selección de las funciones principales observadas en la educación virtual (EV) y que fueron nombradas como áreas primarias (Figura 2).

\section{Figura 2. Selección de áreas primarias en la EV}

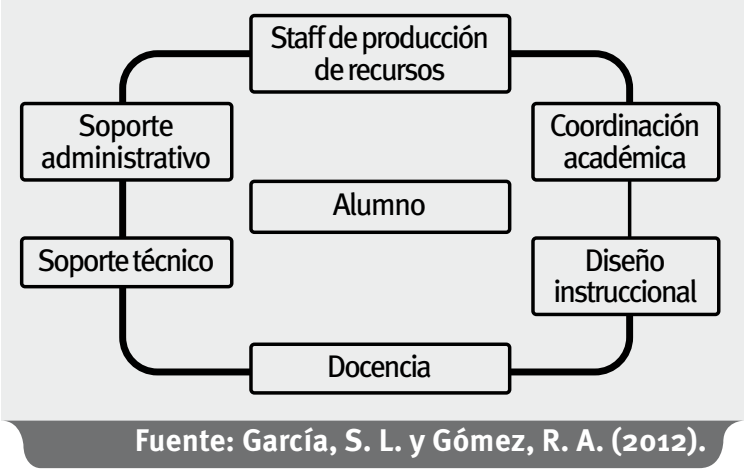

El análisis de los componentes de cada parte de la selección de áreas primarias en la educación virtual, permitió encontrar en ellas elementos coincidentes que condujeron a la determinación de los procesos necesarios comunes en la operación y ejecución de la educación superior virtual. Los procesos necesarios se identificaron en la operación de 7 áreas: 1) preparación de la infraestructura requerida para operar la modalidad dependiendo de su tipología como universidad virtual; 2) la implementación de accesos que condujeran a los usuarios a la interactividad del aula en el ambiente virtual como parte de su asistencia a las clases regulares de su currícula; 3) la preparación oficial de los programas académicos de grado, que incluía desde la obtención de su validez curricular y la especificidad de creación curricular para la modalidad hasta la contratación de la planta docente; 4) la creación y producción de recursos académicos compatibles con la relación enseñanza-aprendizaje en ambientes virtuales; 5) la implementación educativa como la ejecución formal del proceso enseñanza-aprendizaje en el aula, incluyendo la interactividad y comunicación entre el docente tutor y sus alumnos; 6) la preparación para dar atención en servicios escolares; 7) la preparación para ofrecer atención en los procesos administrativos de carácter interno de la IES.

Estos procesos no correspondieron a etapas cronológicamente expuestas, sino a requerimientos que se ejecutan alternada o paralelamente en las diferentes etapas de operación de un programa virtual de grado. 
Al llevar a cabo este análisis para realizar la selección de procesos necesarios, se estimó oportuno que en el estudio se tomaran en cuenta además de las de sub-dimensiones que se encontraban dentro de la dimensión organizativa en la teoría de la gestión escolar, la adición de una sub-dimensión a las dos ya existentes, relacionada con los elementos y procesos de la modalidad en línea; de esta forma, a los sistemas de gestión de servicios educativos y administrativos comunes de un formato académico presencial, se les integra de forma paralela el espacio destinado a la gestión de la virtualidad, lo cual produjo una composición que comprendió tres subdivisiones: la gestión del sistema virtual, la gestión de servicios administrativos y la gestión de servicios educativos.

Cada una de estas tres subdivisiones integró en sí a un grupo de procesos necesarios, por lo que se llegó a la decisión de tomarlos como el referente dimensional de estos, conformando la dimensión organizativa de la gestión escolar propia de la modalidad en línea. La Figura 3 muestra la composición completa de la dimensión organizativa de la gestión escolar remota, donde se observa la relación entre los procesos necesarios del sistema de gestión que requiere una IES de acuerdo con cada una de las tres dimensiones.
Figura 3. Procesos necesarios en la operación de un programa virtual

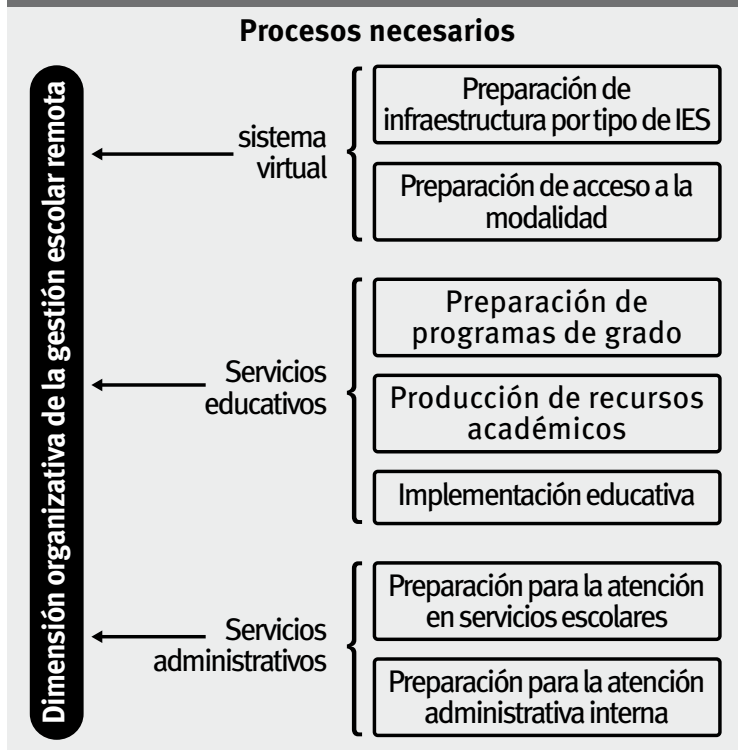

Fuente: García, S. L. y Gómez, R. A. (2012).

\subsection{La determinación de los indicadores por procesos críticos}

Al aplicar las herramientas para la recolección de información y procesamiento de las tablas de registro, se origina un proceso de depuración de los indicadores de los procesos necesarios que dan pie a la construcción de los procesos críticos. La Tabla 2 muestra los elementos que los integran y su reclasificación en los sistemas de gestión escolar remota.

Tabla 2. Elementos que integran a los procesos críticos por sistema

\begin{tabular}{|c|c|c|}
\hline Sistema & Proceso crítico & Elemento \\
\hline \multirow[t]{3}{*}{$\begin{array}{l}\text { Sistema } \\
\text { para la } \\
\text { virtualidad }\end{array}$} & Infraestructura física & $\begin{array}{l}\text { *Tipo de IES virtual } \\
\text { *Instalaciones físicas para atención del usuario virtual } \\
\text { *Apoyo presencial en la modalidad }\end{array}$ \\
\hline & Infraestructura tecnológica & $\begin{array}{l}\text { *Plataforma de gestión de aprendizaje } \\
\text { *Nombre de la plataforma personalizada } \\
\text { *Plataforma para soporte administrativo }\end{array}$ \\
\hline & Infraestructura de acceso & $\begin{array}{l}\text { *Forma de acceso a la modalidad desde el origen } \\
\text { *URL de la IES a través de la cual se accede } \\
\text { *URL de la IES virtual } \\
\text { *Forma de acceso al campus virtual } \\
\text { *Forma de acceso a la información } \\
\text { *Acceso a servicios de apoyo para la modalidad } \\
\text { *Acceso a servicios académicos periféricos } \\
\text { *Medios } \\
\text { *Comunidades virtuales }\end{array}$ \\
\hline \multirow{2}{*}{$\begin{array}{l}\text { Sistema de } \\
\text { servicios } \\
\text { educativos }\end{array}$} & Oferta académica de la modalidad & $\begin{array}{l}\text { *Planes de estudio a nivel licenciatura para oferta virtual } \\
\text { *Autonomía de la oferta virtual }\end{array}$ \\
\hline & Recursos educativos para la virtualidad & $\begin{array}{l}\text { *Bases pedagógicas para la virtualidad } \\
\text { *Apoyo para producción y provisión de recursos }\end{array}$ \\
\hline \multirow{2}{*}{$\begin{array}{l}\text { Sistema de } \\
\text { servicios } \\
\text { administrativo }\end{array}$} & Control escolar & $\begin{array}{l}\text { *Plataforma para control escolar } \\
\text { *Origen primario del servicio de control escolar }\end{array}$ \\
\hline & Servicios internos & $\begin{array}{l}\text { * Servicios administrativos } \\
\text { *Origen primario del servicio administrativo } \\
\text { *Proveedores de servicios externos a disposición del usuario }\end{array}$ \\
\hline
\end{tabular}


Los cambios reflejados en relación con los procesos necesarios fueron que en el sistema para la virtualidad se separó a la categoría de infraestructura en infraestructura física y tecnológica; en el sistema de servicios educativos se integraron la oferta educativa y la regularización de sus programas en relación con la validez oficial y sus requisitos; se eliminaron las categorías que por naturaleza tendían a medir el desempeño en el aula, y para el sistema de servicios administrativos se incrementó un elemento no considerado con anterioridad pero que resultó una presencia constante en la inmersión al ambiente: la información.

\subsection{Resultados por indicadores de los procesos críticos}

Los resultados por indicadores de los procesos críticos se agruparon para su presentación en los tres sistemas de la gestión escolar remota.

\subsubsection{El sistema para la virtualidad}

Los resultados relacionados con la infraestructura física mostraron que respecto al tipo de IES virtual, el $30 \%$ son universidades presenciales que introducen elementos de virtualidad en su dinámica educativa y funcionan con unidades al interior de las instituciones compartiendo currícula, recursos y personal; el $20 \%$ son universidades virtuales con extensión universitaria presencial que funcionan con unidades al interior de las instituciones con distinta organización y currícula; y otro $20 \%$ son universidades virtuales adosadas con la universidad tradicional que funcionan con unidades al interior de las instituciones compartiendo currícula, recursos y personal. El $80 \%$ de las IES tiene estructura física para la presencia de atención, y $20 \%$ da acceso a sus usuarios a través de oficinas descentralizadas; $90 \%$ de las IES presenta algún canal de apoyo presencial para sus usuarios, y se observó que este apoyo tiene que ver con centros de servicio infraestructural, con equipos y sistemas técnicos para acceder a la modalidad; también funcionan como complemento de los programas no presenciales para tutoría directa y servicios escolares.

En el indicador de infraestructura tecnológica se registró que en relación con la plataforma de gestión de aprendizaje, $90 \%$ de las IES seleccionadas utilizan, de forma personalizada y acorde con la identidad corporativa, plataformas basadas en el software libre de Moodle y $10 \%$ utiliza los servicios de los desarrolladores de Blackboard; 90\% de las IES tienen personalizada su plataforma de aprendizaje a través de la asignación de una identidad propia y solo el $10 \%$ integra una identidad de imagen sin agregar un nombre propio que lo distinga en el mercado. Se observó que el $100 \%$ de la muestra utiliza diseños especiales para su plataforma de soporte administrativo, en función de la gama de servicios que ofertan y la forma en que los vinculan y resuelven en la gestión remota.

En relación con la infraestructura de acceso se registró que en el $90 \%$ de los casos se observa que en la página institucional de la IES existen múltiples accesos de ingreso a la modalidad, y en el $70 \%$ los accesos no presentan claridad en su identidad como universidad virtual, sino que aparecen como apéndices de la oferta o como campus virtual adyacente a lo presencial. Con respecto a la autonomía en el acceso al campus virtual como entidad universitaria (URL de la IES virtual), se encontró con que el $90 \%$ de las IES virtuales tienen su propia dirección de acceso directo, adicional al de su ingreso a través del vínculo institucional; en todos los casos la página de acceso virtual fue diseñada con la identidad institucional y se observa la pertenencia. El $10 \%$ de las IES pareciera tener en desuso páginas paralelas como universidad virtual, causando confusión en los motores de búsqueda. En otro $10 \%$ se percibe la ausencia de estructura y la identidad formal que identifique a la universidad virtual como tal, pues sus elementos de acceso muy son muchos, dispersos y de uso divergente.

En relación con los acceso hacia elementos informativos para orientación al usuario los resultados indican que un $90 \%$ explican su misión, visión y objetivos; $80 \%$ muestra calendarios; $70 \%$ presentan sus perfiles, y en el mismo porcentaje sus avisos; $60 \%$ muestran antecedentes y reglamentos; $50 \%$ consideran información para convocatorias, nuevos ciclos y cursos, y el mismo porcentaje incluye un apartado de preguntas frecuentes y becas; $40 \%$ tiene información sobre organización institucional, explicación de modelos y noticias;30\% información de contacto y $20 \%$ costos.

En los indicadores de los elementos de apoyo a la modalidad sobresalió con un $70 \%$ el acceso a software y herramientas de apoyo académico; $60 \%$ consideró cursos propedéuticos y/o de inducción, soporte técnico, manuales y tutoriales; $50 \%$ ofrece apoyos para hacer contacto institucional pero sólo $20 \%$ ofrece contacto para apoyo en tiempo real; $30 \%$ apoya con trámites escolares vía internet y otro $20 \%$ lo hace integrando accesibilidad para capacidades diferentes y acceso directo a recursos y materiales. En la infraestructura de acceso para apoyos complementarios, el $70 \%$ de las IES virtuales utilizan a las bibliotecas virtuales como su principal recurso; $30 \%$ las revistas digitales; $20 \%$ los catálogos y las redes 
de acervos digitales. Las revistas y los boletines como medios impresos digitales representaron el medio más utilizado (80\%), la prensa $60 \%$ y la televisión en línea un $50 \%$ seguido de la radio y los foros con un $30 \%$ y $40 \%$ respectivamente; los podcast, webcast y sitios web representaron $20 \%$ cada uno.

La presencia de las comunidades virtuales se hace patente en los resultados observados, pues el 90\% tiene presencia en Facebook, $60 \%$ en Twitter y 40\% en YouTube; $20 \%$ en Flickr y $10 \%$ en Slideshare. A pesar de que estos porcentajes parecieran revelar una gran actividad por estos medios abiertos, el análisis muestra que la mayoría se ejecuta a partir de los usuarios y no de las instituciones y no evidencian mantenimiento; sólo 4 de las 9 IES que muestran actividad por Facebook tienen presencia formal, oficial y constante en este medio.

\subsubsection{El sistema de servicios educativos}

Los resultados observados en el indicador de la oferta académica de la modalidad arrojaron que un $60 \%$ de la oferta académica virtual depende de la oferta presencial, y $40 \%$ es autónoma.

En relación con los recursos educativos para la virtualidad se tiene que el $50 \%$ presenta un modelo pedagógico desarrollado para la modalidad y un $40 \%$ cuenta con modelo educativo especial para lo virtual; $30 \%$ también señala un modelo tecnológico y otro porcentaje igual manifiesta tener un modelo de aplicación especial en función con la manera de cursar la licenciatura (vr.gr. modular y escalable) y $10 \%$ se ciñe todavía al modelo presencial de la IES tradicional. En las IES donde no se encontraron evidencias sobre la existencia de modelos para la virtualidad puede deberse a que no está explicitado y exhibidos. En relación con los apoyos para la producción y provisión de recursos y materiales, los resultados indican que $50 \%$ de las IES cuentan con un centro de apoyo especializado, $30 \%$ de capital público y $20 \%$ privado; las de origen público cuentan con una infraestructura amplia y de vanguardia investigativa, además de una gama muy variada de opciones para el desarrollo de recursos y materiales. Del otro $50 \%$ no se encontraron evidencias observables.

\subsubsection{El sistema de servicios administrativos}

En el sistema de control escolar 90\% mostró acceso en línea a diversos servicios e información de control escolar, aunque en relación con la forma de ejecutarlos el $50 \%$ otorgaba servicio presencial con procesos parciales en línea y dependía de lo presencial; $20 \%$ atendía de forma institucional y presencial, y $10 \%$ los hacía semivirtual con asistencia presencial y enlazado a la red institucional de servicios; un porcentaje igual lo hace en línea y enlazado en la red institucional de servicios, y otro $10 \%$ otorga el servicio en línea y de manera autónoma.

Para el indicador de servicios internos de las IES el comportamiento del servicio fue igual a lo arrojado por los resultados de la observación de los servicios de control escolar.

\section{Conclusiones}

En México existe una tendencia a la plataformización de la educación superior más que a un crecimiento de la modalidad virtual, lo cual se manifiesta en su dependencia respecto a los sistemas presenciales, $y$ al hecho de que no se obliga a la IES a diferenciar entre una relación con la educación a distancia versus una prestación de servicios académicos a través de la virtualidad.

Los elementos que describen la forma de operación que se lleva a cabo de manera tácita en las IES oferentes de programas virtuales, son parte de 7 procesos críticos agrupados en tres sistemas diferentes: el sistema para la virtualidad, el sistema de servicios educativos y el sistema de servicios administrativos. $\mathrm{Ni}$ el origen de recursos aplicados a la modalidad, ni el tamaño de la IES, afectaron la presencia de los elementos componentes de los procesos críticos, lo que reveló características afines en la operatividad de las IES virtuales, pero ejecutadas a través de herramientas y estructuras educativas diferentes.

Los procesos que resultan críticos para la ejecución de la práctica didáctica y para la medición de desempeño en el aula, no deben ser reglamentados en un modelo de operación administrativa, pues no resultan elementos que deban ser regulados a partir de normas específicas, porque su implementación depende desde los modelos educativos y pedagógicos hasta la libertad de cátedra establecidos en la estrategia académica.

En relación con el sistema para la virtualidad se determinó que en los casos donde se ofertan ambos formatos académicos, la administración de la IES a nivel institucional otorga al programa virtual una identidad universitaria tangible, haciéndolo partícipe de las ventajas y soportes que se otorgan a sus participantes presenciales; la IES también integran en su página principal el inicio de la cadena de navegación hacia lo virtual.

En la plataforma de gestión del conocimiento se integran las aulas virtuales y en la de gestión administrativa las oficinas virtuales; las dos plataformas 
están vinculadas a través del portal institucional y de la entrada principal a la oferta virtual de la IES, pero con accesos diferentes. Los sistemas de comunicación forman una constante en el esquema de operación, y van desde correos electrónicos y mensajería instantánea hasta el desarrollo de comunidades virtuales complementarias.

La tipología de universidad virtual más común en el mercado es la presencial que introduce elementos de virtualidad o deja surgir a una institución paralela dentro de sí, y en ambos casos funcionan con ésta compartiendo recursos, programas y personal, lo que evidencia la propensión a pensar en la virtualidad a través de un esquema presencial; sin embargo, la tendencia que muestra la tercera tipología más común en la operación actual, universidades virtuales con extensiones presenciales, es que la sociedad académica en general se está viendo obligada a crear sistemas virtuales como opción alterna a los mercados emergentes. Y si bien la atención al usuario es primordialmente en línea, los segmentos de mercado que se acercan a la modalidad virtual para los estudios de nivel superior todavía tienden a requerir de apoyos presenciales, sea para cuestiones académicas y escolares pero también para obtención de acceso a equipo de trabajo; la preocupación de cubrir esta necesidad es notoria, sobre todo en las universidades de origen público, a través de instalaciones físicas modulares y nodos. La preferencia para el uso de las plataformas tecnológicas fue por el software libre en lo académico, mientras que lo administrativo tendió hacia una reingeniería que concentra información y recursos en red para su gestión.

Se identificaron 4 grupos de acceso a la modalidad: las páginas de acceso institucional, las de la universidad virtual, los vínculos al campus virtual desde una y otra, las necesidades de información detectadas en el usuario y satisfechas en línea, sobre todo en lo referente a datos sobre la institución, sobre el funcionamiento y características de la modalidad y de los programas de la institución en este formato virtual; se ha detectado la necesidad del usuario de contar con herramientas digitales de uso común, manuales y tutoriales sobre procedimiento y manejo de recursos. La inducción y el soporte técnico son una constante que se introduce desde diferentes ángulos pero siempre está presente como uno de los sellos de apoyo para la modalidad. El acceso a los apoyos complementarios para el ejercicio de la modalidad, se da sobre todo a través de la implementación de sistemas de comunicación paralela a los formales, pero no por ello menos importantes por la dinámica social que las cobija como Facebook, Twitter y YouTube.
En torno al sistema de servicios educativos se determinó que la autonomía de una IES virtual se da en función de la integración de procesos en la estructura de atención remota de sus usuarios y de la creación de oferta educativa específica para su operación a través de la virtualidad, y que a pesar de que la oferta virtual tiende a depender de programas presenciales, un alto porcentaje de participación de planes hechos para funcionar en línea, demuestran que esa situación está en proceso de cambio. Esta autonomía se evidencia ante el crecimiento en la oferta de programas académicos de este tipo, la provisión de elementos y recursos para la práctica académica y la comunicación e interactividad en el aprendizaje-enseñanza de los ambientes virtuales.

Hay una tendencia a implementar el uso de modelos pedagógicos, educativos y tecnológicos especialmente diseñados para el ejercicio de la virtualidad a través de las líneas marcadas por ellos. Para los recursos utilizables hay tres vertientes de adquisición: 1) la selección de materiales ya divulgados y/o publicados formalmente por un tercero; 2 ) la adquisición de derechos de materiales y recursos creados y desarrollados por un tercero pero todavía sin publicar formalmente; 3) la creación y desarrollo de materiales y recursos propios con un equipo facultado y multidisciplinario de la IES, especialmente diseñados para su explotación académica en la modalidad virtual. Los centros de apoyo para este propósito se perciben como desarrollados fuertes y abiertos a los usuarios.

El control escolar y los servicios administrativos internos institucionales son la esencia de los servicios administrativos que distinguen al sector educativo, por ello su funcionamiento a través de la virtualidad no fue tan diferente como en el caso de las dimensiones anteriores; de hecho, su funcionamiento intrínseco al del formato presencial conforma una sensación de pertenencia e identidad institucional, pues se percibe una actuación integral, sin distingos por segmentación de usuarios. Y aunque tanto en los servicios de control escolar como en los administrativos internos de la IES el origen primario del servicio es de orden presencial con procesos parciales en línea y alta dependencia de la institución presencial, la tendencia que se observa sobre la centralización de los datos y recursos otorga una perspectiva de funcionamiento en común entre ambos formatos, lo que habla de integración organizativa.

\section{Limitaciones de la investigación}

Una limitante fue el tamaño de la muestra ante la decisión de no cambiar de instituciones observadas 
cuando se percibía que su desempeño como universidad virtual en realidad no era tal, sino solamente se utilizaba a las plataformas de gestión académica y administrativa como una herramienta para la oferta de sus servicios educativos presenciales. Por ello se considera que para consolidar la tendencia de los resultados y las conclusiones es necesario incrementar la cantidad de universidades observadas.

\section{Nuevas líneas de investigación}

Los resultados y conclusiones de esta investigación son la base para construir un nuevo paradigma especialmente diseñado para la educación superior virtual, dando pie a la creación de normatividad específica para este formato de enseñanza-aprendizaje. Para ello es necesario la continuación de esta investigación con el fin de profundizar sobre el comportamiento de las IES oferentes de estos servicios académicos, encontrando parámetros administrativos que sirvieran de mínimos necesarios para el funcionamiento y operación de la modalidad virtual en la educación de nivel superior en México.

\section{Referencias}

Asociación Nacional de Universidades e Instituciones de Educación Superior (ANUIES). (2001). Plan Maestro de Educación Superior. Recuperado 26/09/2012 de: http://www.anuies.mx/servicios/d_estrategicos/ pdf/plan_maestro_1.pdf

Asociación Nacional de Universidades e Instituciones de Educación Superior (ANUIES). (2003). Elementos normativos a considerar para los programas de educación superior impartidos en modalidades alternativas a la escolarizada. Recuperado 01/05/2011 de: http://www.anuies.mx/e_proyectos/html/estrategicos1.html

Asociación Nacional de Universidades e Instituciones de Educación Superior (ANUIES). (2007). Ampliación de la oferta de nuevos programas, y apoyo al fortalecimiento de la misma mediante modalidades no presenciales o mixtas. Conclusiones de participación en foro. México, D.F., México: Secretaría de Educación Pública, Subsecretaría de Educación Superior.

Chan, M.E. (2010). La comunicación como mediación entre la tecnificación y la virtualización de las instituciones educativas. Mediaciones Sociales, (6), (pp. 65-89).

Flores, M.M. (2009). Propuesta de normatividad para asesores de Programas Educativos en Línea en la Universidad de Guadalajara. Recuperado
01/05/2011 de: http://bdistancia.ecoesad.org.mx/ cont/Secciones/NumConsto3/ReflexionesMF.html

García, L. (2008). Historia de la Educación a Distancia. Recuperado 20/10/2008 de: http://www.utpl.edu. ec/ried/images/pdfs/vol2-1/historia.pdf

Lemke, J. (1993). Education, cyberspace and change. Recuperado 26/09/2012 de: http://serials.infomotions.com/aejvc/aejvc-v1no1-lemke-education.txt

Lévy, P. (1999). ¿Qué es lo virtual? Diego Levis, (Trad.) Barcelona, España: Paidós Ibérica S.A.

López, C. (2009). Hacia la construcción de un marco legal y normativo de la Educación a Distancia en México. Recuperado 01/05/2011 de: http:// bdistancia.ecoesad.org.mx/cont/Secciones/ NumConsto3/Resena.html

Rodríguez, R. (2006). La polémica sobre las universidades patito y las negociaciones SEP-FIMPES en torno al RVOE. Recuperado 01/05/2011 de: http://www.ses. unam.mx/publicaciones/articulos.php?idart $=278$

Rodríguez, R. (2006a). Relaciones peligrosas. Primera parte. La polémica sobre las universidades patito y las negociaciones SEP-FIMPES en torno al RVOE. Recuperado 27/09/2012 de: http://www.ses. unam. $\mathrm{mx} /$ publicaciones/articulos.php?idart $=\mathbf{2 7 8}$

Sánchez, J. A. (2010). Cuerpo y tecnología. La virtualidad como espacio de acción contemporánea. Argumentos (Méx.), 23, (p. 62).

Silvio, J. (1998). La virtualización de la educación superior: alcances, posibilidades y limitaciones. Educación Superior y Sociedad, 2, (p. 1).

Silvio, J. (2006). Hacia una educación virtual de calidad, pero con equidad y pertinencia. RU\&SC. Revista de Universidad y Sociedad del Conocimiento, 3, (p. 001).

Tejeida, R. (06/2003). Modelo de Planeación para el sistema Educación con Ambiente Virtual del Instituto Politécnico Nacional. Tesis doctoral, Instituto Politécnico Nacional, SEPI ESCA, México, D.F, México.

UNESCO. (1998). La educación superior en el siglo XXI: visión y acción. Recuperado 27/09/2012 de: http:// www.unesco.org/education/educprog/wche/ declaration_spa.htm

UNESCO. (2009). Asociación Internacional de Universidades. 08/03/2009, de: http://www.unesco.org/ iau/ 